\title{
Medical Image of the Week: Stomach Rupture
}

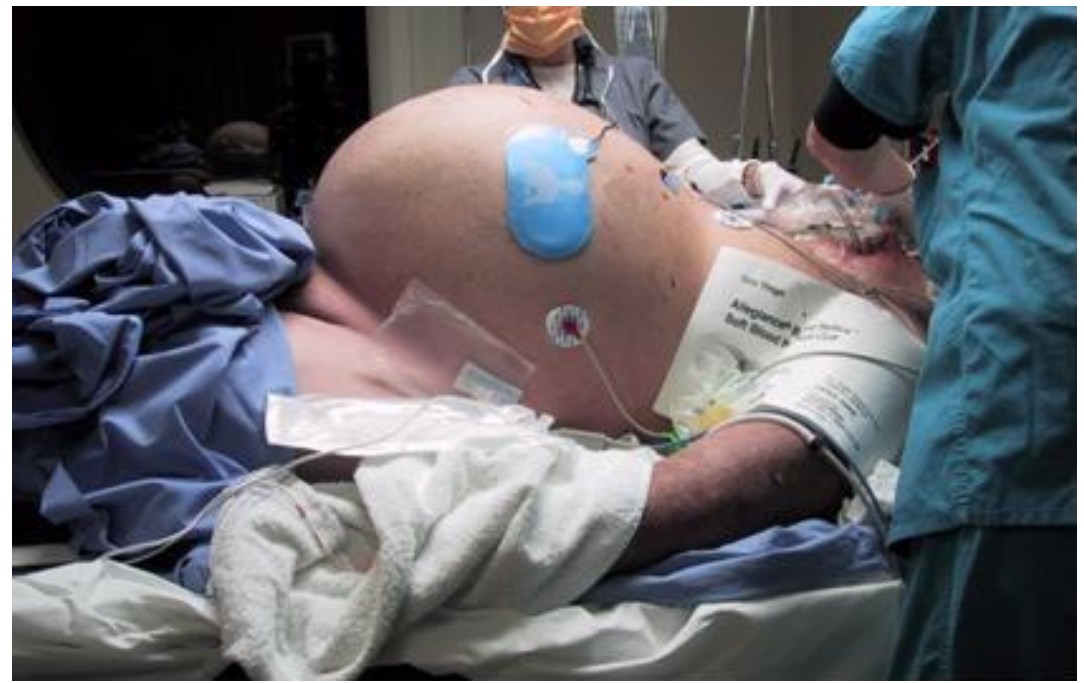

Figure 1. Cross table view of patient showing massively dilated abdomen.

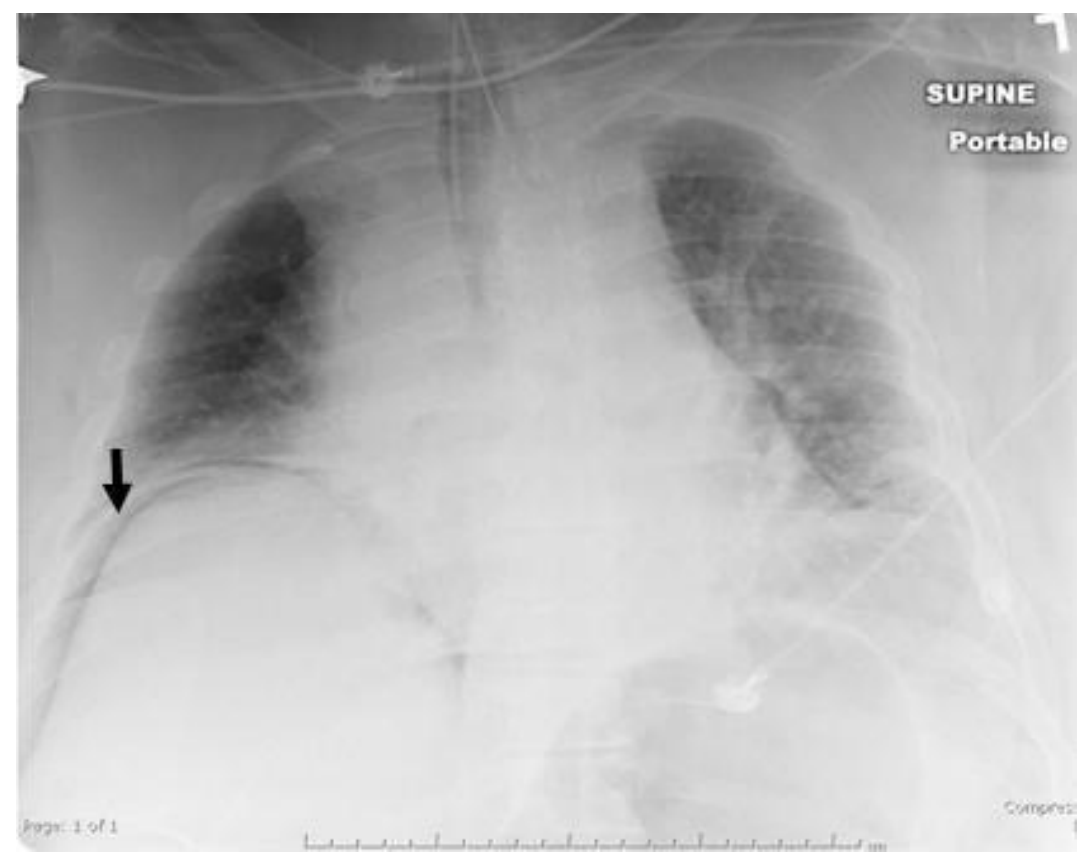

Figure 2. Chest x-ray showing air under diaphragm (arrow).

A 61-year-old man was transferred from another hospital for further care. He had a history of oxygen-dependent chronic obstructive pulmonary disease in addition to congestive heart failure, hypertension and diabetes mellitus. He had been seen earlier in the day at his primary care physician's office for a routine visit. Although he was asymptomatic, emergency medical services (EMS) were called 
because of significant hypoxemia detected by pulse oximetry. EMS noted that the patient said he "feels OK". However, a decision was made to intubate the patient. Multiple failed intubation attempts failed and he suffered a cardiopulmonary arrest. He was successfully resuscitated and underwent a cricotracheotomy with an uncuffed endotracheal tube. When transferred his mouth was taped shut and his nose clamped. His abdomen was markedly distended and tympanic (Figure 1). A supine chest x-ray showed air under the diaphragm. Abdominal exploration showed a ruptured stomach which was repaired. He made an uneventful recovery.

The difficult airway outside the operating room can be problematic. While preparation for airway control are made, preoxygenation should be performed (1). The patient should be placed in the "sniffing" position and mask ventilation performed. Appropriate positioning - with the tragus of the ear elevated parallel to the sternum - may require special preparation in obese patients. When adequate preoxygenation is accomplished endotracheal intubation can be attempted. However, when endotracheal intubation fails and/or mask ventilation is inadequate a variety of advanced intubation techniques can be considered including a laryngeal mask airway, fiberoptic intubation, cricothyroidotomy, or transtracheal jet ventilation (1).

Confirmation of proper endotracheal tube placement should be completed in all patients (2). Physical examination methods such as auscultation of chest and epigastrium, visualization of thoracic movement, and fogging in the tube are not sufficiently reliable to confirm endotracheal tube placement. During intubation, direct visualization of the endotracheal tube passing through the vocal cords into the trachea, especially with the use of a videolaryngoscope, constitutes firm evidence of correct tube placement. Use of an end-tidal carbon dioxide detector (i.e., continuous waveform capnography, colorimetric and non-waveform capnography) to evaluate and confirm endotracheal tube position should be performed. For patients in cardiac arrest and for those with markedly decreased perfusion other methods of confirmation such as an esophageal detector device, ultrasound, or bronchoscopy should be used.

Robert A. Raschke, MD

University of Arizona College of Medicine Phoenix

Phoenix, AZ USA

\section{References}

1. Langeron $\mathrm{O}$, Amour J, Vivien B, Aubrun F. Clinical review: management of difficult airways. Crit Care. 2006;10(6):243. [CrossRef] [PubMed]

2. American College of Emergency Physicians. Verification of endotracheal tube placement. January 2016. Available at: https://www.acep.org/Clinical--Practice-Management/Verification-of-Endotracheal-TubePlacement/\#sm.00004sk8v7vduedxxs618zbgnij0n (accessed 1/24/18). 